\title{
COLONIZATION AND COLLAPSE ON HOMOGENEOUS TREES
}

\author{
VALDIVINO V. JUNIOR, FÁBIO P. MACHADO, AND ALEJANDRO ROLDÁN-CORREA
}

\begin{abstract}
We investigate a basic immigration process where colonies grow, during a random time, according to a general counting process until collapse. Upon collapse a random amount of individuals survive. These survivors try independently establishing new colonies at neighbour sites. Here we consider this general process subject to two schemes, Poisson growth with geometric catastrophe and Yule growth with binomial catastrophe. Independent of everything else colonies growth, during an exponential time, as a Poisson (or Yule) process and right after that exponential time their size is reduced according to geometric (or binomial) law. Each survivor tries independently, to start a new colony at a neighbour site of a homogeneous tree. That colony will thrive until its collapse, and so on. We study conditions on the set of parameters for these processes to survive, present relevant bounds for the probability of survival, for the number of vertices that were colonized and for the reach of the colonies compared to the starting point.
\end{abstract}

\section{INTRODUCTION}

Biological populations are subject to disasters that can cause from a partial elimination of the individuals until their total extinction. When a disaster occurs surviving individuals may react in different ways. A strategy adopted by some populations is the dispersion. In this case, individuals migrate, trying to create new colonies in other locations, there may be competition or collaboration between individuals of the same colony. Once they settle down a new colony in a new spot, again another disaster can strike, which causes a new collapse.

In this type of population dynamics there are some issues to consider, such as: What is the duration of colonization until the moment of the disaster? How much the population grows until be hit? How many individuals will survive? How survivors react when facing a disaster?

In recent articles, the main variables considered in population modeling are (i) the spatial structure where the colonies are located and individuals can move, (ii) the lifetime of a colony until the moment of collapse, (iii) the evolution of the number of individuals in the colony (random or deterministic growth, possible deaths or migration), (iv) the way the cathastrophes affects the size of the colony allowing or not the survival of some

Date: November 5, 2018.

2010 Mathematics Subject Classification. 60J80, 60J85, 92D25.

Key words and phrases. Branching processes, Coupling, Catastrophes, Population dynamics.

Research supported by CNPq (306927/2007-1), FAPESP (2010/50884-4) and Universidad de Antioquia (SUI XXXXX).. 
individuals and $(\mathrm{v})$ whether the individuals that survive to the catastrophe are able to spread out.

Brockwell et al. [5] and later Artalejo et al. [1] considered a model for the growth of a population subject to collapse. In their model, two types of effects when a disaster strikes are analyzed separately, binomial effect and geometric effect. After the collapse, the survivors remain together in the same colony (there is no dispersion). They carried out an extensive analysis including first extinction time, number of individuals removed, survival time of a tagged individual, and maximum population size reached between two consecutive extinctions.

More recently, Schinazi [12] and Machado et al. [11] proposed stochastic models for this kind of population dynamics. For these models they concluded that dispersion is a good survival strategy. Latter Junior et al. [10] showed nice combinations of a type of catastrophe, spatial restriction and individual survival probability when facing the catastrophe where dispersion may not be a good strategy for group survival. For a comprehensive literature overview and motivation see Kapodistria et al. [7].

The paper is divided into four sections. In Section 2 we present a general model for the growth of populations subject to collapses, introduce the variables of interest, notation and two particular schemes: Poisson growth with geometric catastrophe and Yule growth with binomial catastrophe. In Section 3 we present the main results of the paper while their proofs are in Section 4.

\section{Colonization and Collapse models}

In the beginning all vertices of $\mathbb{G}$, a infinite conected graph, are empty except for the origin where there is one individual. Besides that, at any time each colony is started by a single individual. The number of individuals in each colony behaves as $\mathcal{C}$, a Counting Process. To each colony is associated a non-negative random variable $T$ which defines its lifetime. After a period of time $T$, that colony collapses and the vertex where it is placed becomes empty. At the time of collapse, with a random effect $\mathcal{E}$, some individuals in the colony are able to survive while others die. By simplicity we represent this quantity by $N$. Note that this random quantity depends on the Counting Process which defines the growth of the colony, on the distribution of $T$ and on how the collapse afects the group of individuals present in the colony at time $T$. Each one individual that survives ( $N$ individuals) tries to found a new colony on one of the nearest neighbour vertices by first picking one of them at random. If the chosen vertex is occupied, that individual dies, otherwise the individual founds there a new colony. We denote the Colonization and Collapse model generally described here either by $\{\mathbb{G} ; N\}$ or $\{\mathbb{G} ; \mathcal{C}, \mathcal{E}, T\}$, a stochastic process whose state space is $\mathbb{N}^{T^{d}}$. Along this paper we concentrate our attention on $\mathbb{T}^{d}$, a homogeneous tree where every vertex has $d+1$ nearest neighbours and on $\mathbb{T}_{+}^{d}$, a tree whose only difference from $\mathbb{T}^{d}$ is that its origin has degree $d$.

Definition 2.1. Let us consider the following random variables

- $I_{d}$ : the number of colonies created from the beginning to the end of the process;

- $M_{d}$ : the distance from the origin to the furthest vertex where a colony is created; 
- $\left\{X_{t}\right\}_{0 \leq t \leq T}$ growth process for the amount of individuals in a colony.

We work in details some specific cases.

- $T$ : Lifetime of a colony

$-T \sim \mathcal{E} x p(1)$, Exponential with mean 1

$$
P[T<t]=1-e^{-t}, t>0
$$

- $X_{t}$ : Growth of the number of individuals

$-X_{t} \sim \mathcal{P}$ oisson $(\lambda t)$, a Poisson point process with rate $\lambda$

$$
P\left[X_{t}=k\right]=\frac{e^{-\lambda} \lambda^{k-1}}{(k-1) !}, k \in\{1,2, \ldots\}
$$

$-X_{t} \sim \mathcal{G}$ eom $\left(e^{-\lambda t}\right)$, a Yule process with rate $\lambda$

$$
P\left[X_{t}=k\right]=e^{-\lambda t}\left(1-e^{-\lambda t}\right)^{k-1}, k \in\{1,2, \ldots\}
$$

- $N$ : Number of individuals able to survive

$-N \mid X_{T} \sim \mathcal{B}\left(X_{T}, p\right)$, Binomial catastrophe

$$
\begin{array}{r}
P\left[N=m \mid X_{T}=k\right]=\left(\begin{array}{c}
k \\
m
\end{array}\right) p^{m}(1-p)^{k-m}, m \in\{0,1, \ldots, k\} \\
-N \mid X_{T}=X_{T}-\min \left\{\mathcal{G} e o m(p)-1 ; X_{T}\right\} \sim \mathcal{G}_{X_{T}}(p), \text { Geometric catastrophe } \\
P\left[N=m \mid X_{T}\right]= \begin{cases}p(1-p)^{X_{T}-m} & \text { if } m \in\left\{1, \ldots, X_{T}\right\} \\
(1-p)^{X_{T}} & \text { if } m=0\end{cases}
\end{array}
$$

In general it is true that

$$
\begin{gathered}
\mathbb{P}(N=n)=\int_{0}^{\infty} \mathbb{P}(N=n \mid T=t) f_{T}(t) d t \\
\mathbb{P}(N=n \mid T=t)=\sum_{x=n}^{\infty} \mathbb{P}\left(X_{T}=x \mid T=t\right) \mathbb{P}\left(N=n \mid X_{T}=x ; T=t\right) .
\end{gathered}
$$

Suppose that individuals are born following a Poisson process at rate $\lambda$, that the collapse time follows an exponential random variable with average $1(T \sim \mathcal{E} x p(1))$ and the individuals are exposed to the collapse effects, one by one, until the first individual survive, if any, then the collapse effects stop. If the collapse effects reach a fixed individual, it survives with probability $p$, meaning that $N_{T} \sim \mathcal{G}_{X_{T}}(p)$ (Geometric catastrophe) or $\mathcal{G}(p)$ for short. Let us consider the distribution of the number of survivals at collapse times

$$
\mathbb{P}(N=0)=\int_{0}^{\infty} e^{-t} \sum_{j=0}^{\infty} \frac{e^{-\lambda t}(\lambda t)^{j}}{j !}(1-p)^{j+1} d t=\frac{1-p}{1+\lambda p}
$$

and for $n \geq 1$ :

$$
\mathbb{P}(N=n)=\int_{0}^{\infty} e^{-t} \sum_{j=n-1}^{\infty} \frac{e^{-\lambda t}(\lambda t)^{j}}{j !} p(1-p)^{j+1-n} d t=\left(\frac{\lambda}{\lambda+1}\right)^{n-1} \frac{p}{\lambda p+1} .
$$


In this case the probability generating function is of $N$ is

$$
\begin{aligned}
\mathbb{E}\left(s^{N}\right) & =\frac{1-p}{1+\lambda p}+\sum_{n=1}^{\infty} s^{n}\left(\frac{\lambda}{1+\lambda}\right)^{n-1}\left(\frac{p}{\lambda p+1}\right) \\
& =\frac{1}{\lambda p+1}\left[1-p+\frac{(\lambda+1) p s}{1+\lambda-\lambda s}\right] .
\end{aligned}
$$

while its average is $\mathbb{E}(N)=\frac{p(\lambda+1)^{2}}{(\lambda p+1)}$.

Suppose now that individuals are born following a Yule process at rate $\lambda$, that $T \sim$ $\mathcal{E} x p(1)$ and that the disaster reach the individuals simultaneously and independently of everything else. Assuming that each individual survives with probability $p$, we have that $N_{T} \sim \mathcal{B}\left(X_{T}, p\right)$ (Binomial catastrophe) or $\mathcal{B}(p)$ for short. Let us consider the distribution of the number of survivals at collapse times.

$$
\begin{aligned}
\mathbb{P}(N=0) & =\int_{0}^{\infty} e^{-t} \sum_{j=1}^{\infty} e^{-\lambda t}\left(1-e^{-\lambda t}\right)^{j-1}(1-p)^{j} d t \\
& =\frac{1-p}{\lambda+1}{ }_{2} F_{1}\left(1,1 ; 2+\frac{1}{\lambda} ; 1-p\right)
\end{aligned}
$$

and for $n \geq 1$

$$
\begin{aligned}
\mathbb{P}(N=n) & =\int_{0}^{\infty} e^{-t} \sum_{j=n}^{\infty} e^{-\lambda t}\left(1-e^{-\lambda t}\right)^{j-1}\left(\begin{array}{c}
j \\
n
\end{array}\right) p^{n}(1-p)^{j-n} d t \\
& =\frac{p^{k}}{\lambda} B\left(k, 1+\frac{1}{\lambda}\right){ }_{2} F_{1}\left(k+1, k ; k+1+\frac{1}{\lambda} ; 1-p\right) .
\end{aligned}
$$

In this setup the probability generating function of $N$ is

$$
\begin{aligned}
\mathbb{E}\left(s^{N}\right) & =\sum_{n=0}^{\infty} s^{n} \int_{0}^{\infty} e^{-t} \sum_{k=n \vee 1}^{\infty} e^{-\lambda t}\left(1-e^{-\lambda t}\right)^{k-1}\left(\begin{array}{l}
k \\
n
\end{array}\right) p^{n}(1-p)^{k-n} d t \\
& =\frac{p s+1-p}{\lambda+1}{ }_{2} F_{1}\left(1,1 ; 2+\frac{1}{\lambda} ; p(s-1)+1\right)
\end{aligned}
$$

and its average is

$$
\mathbb{E}(N)=\left\{\begin{array}{cl}
\frac{p}{1-\lambda} & , \text { se } \lambda<1 \\
\infty & , \text { se } \lambda \geq 1
\end{array}\right.
$$

\section{Main Results}

$\left\{\mathbb{T}^{d}, \mathcal{C}, \mathcal{E}, T\right\}$ is a stochastic process whose state space is $\mathbb{N}^{\mathbb{T}^{d}}$ and whose evolution (status at time $t$ ) is denoted by $\eta_{t}$. For a vertex $x \in \mathbb{T}^{d},\left\{\eta_{t}(x)=i\right\}$ means that at the time $t$ there are $i$ individuals at the vertex $x$. We consider $\left|\eta_{t}\right|=\sum_{x \in \mathbb{T}^{d}} \eta_{t}(x)$. 


\subsection{Phase Transition.}

Definition 3.1. Let $\eta_{t}$ be the process $\left\{\mathbb{T}^{d} ; \mathcal{C}, \mathcal{E}, T\right\}$. Let us define the event

$$
V_{d}=\left\{\left|\eta_{t}\right|>0, \text { for all } t \geq 0\right\} \text {. }
$$

If $\mathbb{P}\left(V_{d}\right)>0$ we say that the process $\left\{\mathbb{T}^{d} ; \mathcal{C}, \mathcal{E}, T\right\}$ survives. Otherwise, we say that the process $\left\{\mathbb{T}^{d} ; \mathcal{C}, \mathcal{E}, T\right\}$ dies out .

Theorem 3.2. Consider the process $\left\{\mathbb{T}^{d} ; N\right\}$. Then $\mathbb{P}\left(V_{d}\right)=0$ if

$$
\mathbb{E}\left[\left(\frac{d}{d+1}\right)^{N}\right] \geq \frac{d}{d+1}
$$

and $\mathbb{P}\left(V_{d}\right)>0$ if

$$
\mathbb{E}\left[\left(\frac{d}{d+1}\right)^{N}\right]<\frac{d-1}{d} .
$$

Corollary 3.3. Consider the process $\left\{\mathbb{T}^{d} ; \mathcal{P}(\lambda), \mathcal{G}(p)\right\}$.

(i) $\mathbb{P}\left(V_{d}\right)=0$ if

$$
\left(\lambda^{2} d+\lambda d+\lambda+d+1\right) p \leq \lambda+d+1
$$

(ii) $\mathbb{P}\left(V_{d}\right)>0$ if

$$
\left(\lambda^{2} d-\lambda^{2}+\lambda d-\lambda+d\right) p>\lambda+d+1 .
$$

Corollary 3.4. Consider the process $\left\{\mathbb{T}^{d} ; \mathcal{Y}(\lambda), \mathcal{B}(p)\right\}$.

(i) $\mathbb{P}\left(V_{d}\right)=0$ if

$$
{ }_{2} F_{1}\left(1,1 ; 2+\frac{1}{\lambda} ; \frac{d(1-p)+1}{d+1}\right) \geq \frac{d(\lambda+1)}{d+1-p} .
$$

(ii) $\mathbb{P}\left(V_{d}\right)>0$ if

$$
{ }_{2} F_{1}\left(1,1 ; 2+\frac{1}{\lambda} ; \frac{d(1-p)+1}{d+1}\right)<\frac{\left(d^{2}-1\right)(\lambda+1)}{d(d+1-p)} .
$$

Observe that for the process $\{\mathbb{G} ; \mathcal{C}, \mathcal{E}, T\}$, when $\mathcal{C} \in\{\mathcal{Y}(\lambda), \mathcal{P}(\lambda)\}$ and $\mathcal{E} \in\{\mathcal{G}(p), \mathcal{B}(p)\}$, by a coupling argument one can see that $\mathbb{P}\left(V_{d}\right)$ is a non-decreasing function of $\lambda$ and also of $p$. Moreover, the function $\lambda_{c}(p)$, defined by

$$
\lambda_{c}(p):=\inf \left\{\lambda: \mathbb{P}\left(V_{d}\right)>0\right\},
$$

is a non-increasing function of $p$, with $\lambda_{c}(1)=0$ and $\lambda_{c}(0)=\infty$.

Definition 3.5. Let $\eta_{t}$ be a $\{\mathbb{G} ; \mathcal{C}, \mathcal{E}, T\}$ for $\mathcal{C} \in\{\mathcal{Y}(\lambda), \mathcal{P}(\lambda)\}$ and $\mathcal{E} \in\{\mathcal{G}(p), \mathcal{B}(p)\}$, with $0<p<1$. We say that $\eta_{t}$ exhibits phase transition on $\lambda$ if $0<\lambda_{c}(p)<\infty$.

Machado et al.(2016) proved phase transition on $\lambda$ for the process $\left\{\mathbb{T}^{d} ; \mathcal{Y}(\lambda), \mathcal{B}(p)\right\}$. So, there exists a function $\lambda_{c}(\cdot):(0,1) \rightarrow \mathbb{R}^{+}$whose graphic splits the parametric space $\lambda \times p$ into two regions. For those values of $(\lambda, p)$ above the curve $\lambda_{c}(p)$, there is survival in $\left\{\mathbb{T}^{d} ; \mathcal{Y}(\lambda), \mathcal{B}(p)\right\}$ with positive probability. Moreover, for those values of $(\lambda, p)$ below the curve $\lambda_{c}(p)$ extinction occurs in $\left\{\mathbb{T}^{d} ; \mathcal{Y}(\lambda), \mathcal{B}(p)\right\}$ with probability 1. 
However, it is not known anything about the continuity and strict monotonicity (in $p$ ) of the function $\lambda_{c}(p)$. If there is continuity and strict monotonicity, then the process also has phase transition in $p$ for each $\lambda \in(0, \infty)$ fixed.

In order to answer the question about phase transition on $p$ for the process $\{\mathbb{G} ; \mathcal{C}, \mathcal{E}, T\}$, when $\mathcal{C} \in\{\mathcal{Y}(\lambda), \mathcal{P}(\lambda)\}$ and $\mathcal{E} \in\{\mathcal{G}(p), \mathcal{B}(p)\}$, we start with the following definition

$$
p_{c}(\lambda):=\inf \left\{p: \mathbb{P}\left(V_{d}\right)>0\right\} \text {. }
$$

Definition 3.6. Let $\eta_{t}$ be a $\{\mathbb{G} ; \mathcal{C}, \mathcal{E}, T\}$ for $\mathcal{C} \in\{\mathcal{Y}(\lambda), \mathcal{P}(\lambda)\}$ and $\mathcal{E} \in\{\mathcal{G}(p), \mathcal{B}(p)\}$, with $\lambda \in(0, \infty)$ fixed. We say that $\eta_{t}$ exhibits phase transition on $p$ if $0<p_{c}(\lambda)<1$.

The item $(i)$ of Corollary 3.4 coincides with item (iii) of Theorem 3.1 from Machado et al. [11. The novelty of Corollary 3.4 is its item $(i i)$ which provides a suficient condition for survival. Corollary 3.4 guarantees phase transition in $p$ for $\left\{\mathbb{T}^{d} ; \mathcal{Y}(\lambda), \mathcal{B}(p)\right\}$ for $\lambda$ large enough, and gives lower and upper bounds for $\lambda_{c}(p)$.

Example 3.7. Consider $\left\{\mathbb{T}^{4} ; \mathcal{Y}(\lambda), \mathcal{B}(p)\right\}$. The equalities in 3.3 and 3.4 provide lower and upper bounds, respectively, for $\lambda_{c}(p)$. See Figure 1. These bounds guarantees phase transition in $p$ for $\lambda>\lambda_{4}^{*}$. Where $\lambda_{d}^{*}$ is an upper bound for $\lim _{p \rightarrow 1^{-}} \lambda_{c}(p)$, where the former is the solution for

$$
{ }_{2} F_{1}\left(1,1 ; 2+\frac{1}{\lambda} ; \frac{1}{d+1}\right)=\frac{\left(d^{2}-1\right)(\lambda+1)}{d^{2}},
$$

see Corollary $3.4(i i)$. The following table shows computations for $\lambda_{d}^{*}$ for some values of $d$

\begin{tabular}{|l|c|c|c|c|c|c|}
\hline$d$ & 2 & 3 & 4 & 5 & 6 & 10 \\
\hline$\lambda_{d}^{*}$ & 0.4555826 & 0.1613016 & 0.08212601 & 0.04961835 & 0.03315455 & 0.01110147 \\
\hline
\end{tabular}

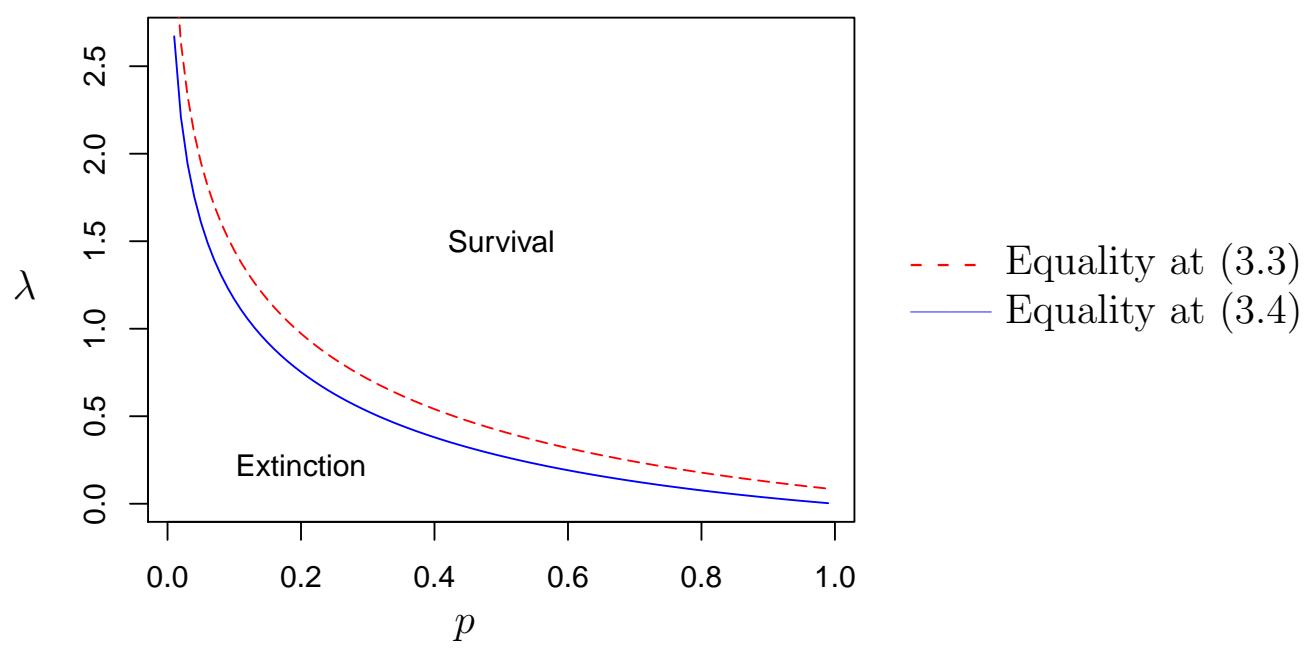

FiguRE 1. Lower and upper bounds for $\lambda_{c}(p)$ in $\left\{\mathbb{T}^{4} ; \mathcal{Y}(\lambda), \mathcal{B}(p)\right\}$ 
Example 3.8. Consider $\left\{\mathbb{T}^{4} ; \mathcal{P}(\lambda), \mathcal{G}(p)\right\}$. The equalities in 3.1 and 3.2 provide lower and upper bounds, respectively, for $\lambda_{c}(p)$. See Figure 2. These bounds guarantees phase transition in $p$ for $\lambda>\lambda_{4}^{*}$. Where $\lambda_{d}^{*}$ is an upper bound for $\lim _{p \rightarrow 1^{-}} \lambda_{c}(p)$, where the former is the solution for

$$
\left(\lambda^{2} d-\lambda^{2}+\lambda d-\lambda+d\right) p=\lambda+d+1,
$$

when $p=1$, see Corollary 3.3 (ii). Thus,

$$
\lambda_{d}^{*}=\frac{1}{d-1}
$$

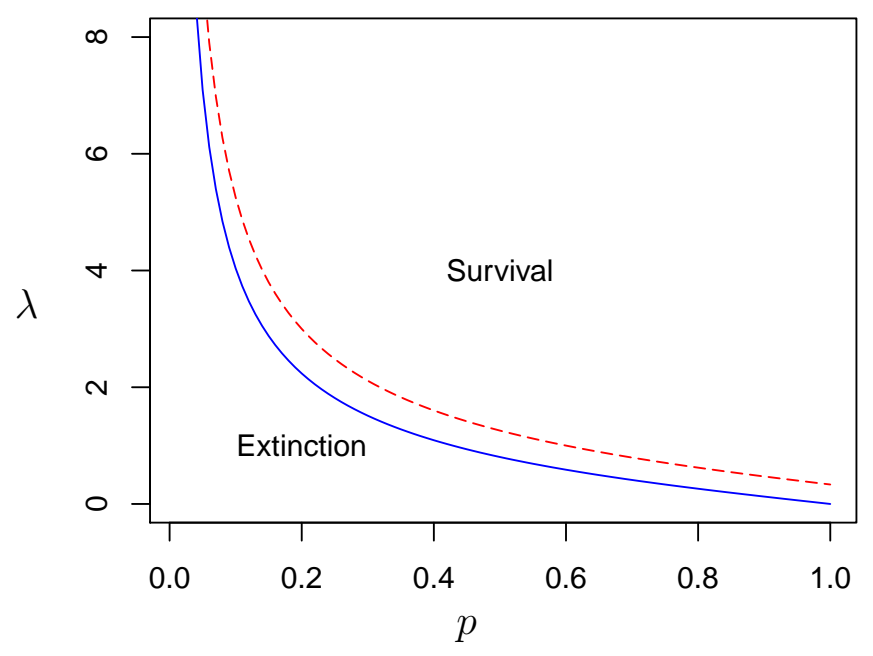

- - Equality at 3.1

Equality at $(\overline{3.2})$

FiguRE 2. Lower and upper bounds for $\lambda_{c}(p)$ in $\left\{\mathbb{T}^{4} ; \mathcal{P}(\lambda), \mathcal{G}(p)\right\}$

3.2. Probability of Survival. We denote by $T(n, k)$ the number of surjective functions $f: A \rightarrow B$, where $|A|=n$ and $|B|=k$, whose value is given, by the inclusion-exclusion principle (see Tucker [15] p. 319), by

$$
T(n, k)=\sum_{i=0}^{k}\left[(-1)^{i}\left(\begin{array}{l}
k \\
i
\end{array}\right)(k-i)^{n}\right], n \geq k .
$$

Theorem 3.9. Consider the process $\left\{\mathbb{T}^{d} ; N\right\}$. We have that

$$
\sum_{r=1}^{d+1}\left[\left(1-\rho^{r}\right)\left(\begin{array}{c}
d+1 \\
r
\end{array}\right) \sum_{n=r}^{\infty} \frac{T(n, r)}{(d+1)^{n}} \mathbb{P}(N=n)\right] \leq \mathbb{P}\left(V_{d}\right) \leq 1-\psi
$$


where $\psi$ and $\rho$ are, respectively, the smallest non-negative solutions of

$$
\begin{aligned}
& \sum_{y=0}^{d+1}\left[s^{y}\left(\begin{array}{c}
d+1 \\
y
\end{array}\right) \sum_{n=y}^{\infty} \frac{T(n, y)}{(d+1)^{n}} \mathbb{P}(N=n)\right]=s, \\
& \sum_{y=1}^{d}\left[s^{y}\left(\begin{array}{l}
d \\
y
\end{array}\right) \sum_{n=y}^{\infty} \frac{T(n, y)+T(n, y+1)}{(d+1)^{n}} \mathbb{P}(N=n)\right]=s-\sum_{n=0}^{\infty} \frac{\mathbb{P}(N=n)}{(d+1)^{n}} .
\end{aligned}
$$

Theorem 3.10. Consider the process $\left\{\mathbb{T}^{d} ; N\right\}$. We have that

$$
\lim _{d \rightarrow \infty} \mathbb{P}\left(V_{d}\right)=1-\nu
$$

where $\nu$ is the smallest non-negative solution of $\mathbb{E}\left(s^{N}\right)=s$.

Corollary 3.11. Consider the process $\left\{\mathbb{T}^{d} ; \mathcal{P}(\lambda), \mathcal{G}(p)\right\}$. Then

$$
\lim _{d \rightarrow \infty} \mathbb{P}\left(V_{d}\right)=\max \left\{0, \frac{p\left(\lambda^{2}+\lambda+1\right)}{\lambda(1+\lambda p)}\right\} .
$$

Example 3.12. Consider the process $\left\{\mathbb{T}^{d} ; \mathcal{P}(5), \mathcal{G}(0.6)\right\}$. If $d=10$ then

$$
\mathbb{P}(N=n)= \begin{cases}\frac{9}{50}\left(\frac{5}{6}\right)^{n}, & n \geq 1 ; \\ \frac{1}{10}, & n=0 .\end{cases}
$$

By using Theorem 3.9 we have that $\psi=0.12226$ and $\rho=0.143256$. Then

$$
0.8733 \leq \mathbb{P}\left(V_{10}\right) \leq 0.8778 .
$$

Besides

$$
\lim _{d \rightarrow \infty} \mathbb{P}\left(V_{d}\right)=0.93
$$

Corollary 3.13. Consider the process $\left\{\mathbb{T}^{d} ; \mathcal{Y}(\lambda), \mathcal{B}(p)\right\}$. Then

$$
\lim _{d \rightarrow \infty} \mathbb{P}\left(V_{d}\right)=1-\nu
$$

where $\nu$ is the smallest non-negative solution of

$$
{ }_{2} F_{1}\left(1,1 ; 2+\frac{1}{\lambda} ; p(s-1)+1\right)=\frac{s(\lambda+1)}{p(s+1)} .
$$

Example 3.14. Consider the process $\left\{\mathbb{T}^{d} ; \mathcal{Y}(\lambda), \mathcal{B}(p)\right\}$. If $\lambda=2$ and $p=0.5$ then, by using Corolary 3.13

$$
\lim _{d \rightarrow \infty} \mathbb{P}\left(V_{d}\right)=0.680977
$$

3.3. The reach of the process. In order to show results for the reach of the process, meaning the distance from the origin to the furthest vertex where a colony is created, let us define a few technical quantities 


\section{Definition 3.15.}

$$
\begin{aligned}
& \alpha=d\left[1-\mathbb{E}\left[\left(\frac{d}{d+1}\right)^{N}\right]\right] \\
& \beta=(d+1)\left[1-\mathbb{E}\left[\left(\frac{d}{d+1}\right)^{N}\right]\right]=\alpha+1-\mathbb{E}\left[\left(\frac{d}{d+1}\right)^{N}\right] \\
& D=\max \left\{2 ; \frac{\beta}{\beta-\mathbb{P}(N \neq 0)}\right\} \\
& B=d(d-1)\left[1-2 \mathbb{E}\left(\left(\frac{d}{d+1}\right)^{N}\right)+\mathbb{E}\left(\left(\frac{d-1}{d+1}\right)^{N}\right)\right]
\end{aligned}
$$

Theorem 3.16. Consider the process $\left\{\mathbb{T}^{d} ; N\right\}$. Assume that

$$
\mathbb{E}\left[\left(\frac{d}{d+1}\right)^{N}\right]>\frac{d-1}{d}
$$

We have that

$$
\frac{[1+D(1-\beta)]\left[1-\beta^{m+1}\right]}{1+D(1-\beta)-\beta^{m+1}} \leq \mathbb{P}\left(M_{d} \leq m\right) \leq \frac{\left[1+\frac{\alpha(1-\alpha)}{B}\right]\left(1-\alpha^{m+1}\right)}{1+\frac{\alpha(1-\alpha)}{B}-\alpha^{m+1}}
$$

and

$$
\frac{\alpha^{2}}{2(B+\alpha)}+\alpha(1-\alpha) \frac{\ln \left[1-\frac{\alpha B}{B+\alpha(1-\alpha)}\right]}{B \ln \alpha} \leq \mathbb{E}\left(M_{d}\right) \leq \frac{D \beta}{D+1}+D(1-\beta) \frac{\ln \left[1-\frac{\beta}{1+D(1-\beta)}\right]}{\ln \beta}
$$

Corollary 3.17. Consider the process $\left\{\mathbb{T}^{d} ; \mathcal{P}(\lambda), \mathcal{G}(p)\right\}$. If

$$
\left(\lambda^{2} d+\lambda d+\lambda+d+1\right) p<\lambda+d+1
$$

then Theorem 3.16 holds under the values

$$
\begin{aligned}
& \alpha=\frac{d p(\lambda+1)^{2}}{(d+\lambda+1)(\lambda p+1)}, \beta=\frac{(d+1) p(\lambda+1)^{2}}{(d+\lambda+1)(\lambda p+1)}, D=\max \left\{2 ; \frac{(d+1)(\lambda+1)}{d \lambda}\right\}, \\
& B=2 d(d-1)\left[\frac{(\lambda+1)^{2}(2(\lambda p+1)-1)+(\lambda+1)(p-1) d-(\lambda p+1) d^{2}}{(d+2 \lambda+1)(d+2 \lambda+1)(\lambda p+1)}\right] .
\end{aligned}
$$

Theorem 3.18. Consider the process $\left\{\mathbb{T}^{d} ; N\right\}$. We have that

$$
M_{d} \stackrel{D}{\rightarrow} M
$$

where $\mathbb{P}(M \leq m)=g_{m+1}(0)$, being $g(s)=\mathbb{E}\left(s^{N}\right)$ and $g_{m+1}(s)=g(g(\cdots g(s)) \cdots)$.

Corollary 3.19. Consider the process $\left\{\mathbb{T}^{d} ; \mathcal{P}(\lambda), \mathcal{G}(p)\right\}$. 
(i) If $p \neq\left(\lambda^{2}+\lambda+1\right)^{-1}$ then

$$
\begin{gathered}
\mathbb{P}(M \leq m)=\frac{1-\left(\frac{(\lambda+1)^{2} p}{\lambda p+1}\right)^{m+1}}{1-\frac{\lambda(\lambda p+1)}{(1-p)(\lambda p+1)}\left(\frac{(\lambda+1)^{2} p}{\lambda p+1}\right)^{m+1}}, m \geq 0 \\
\text { and } \\
\mathbb{E}(M)=\frac{\left(1-p\left(\lambda^{2}+\lambda+1\right)\right)}{\lambda(\lambda p+1))} \lim _{s \rightarrow \infty}\left[\psi_{\gamma}\left(1-\frac{\ln \frac{(\lambda+1)(1-p)}{\lambda(\lambda p+1)}}{\ln \gamma}\right)-\psi_{\gamma}\left(s-\frac{\ln \frac{(\lambda+1)(1-p)}{\lambda(\lambda p+1)}}{\ln \gamma}+1\right)\right] \\
\text { where } \gamma=\frac{(\lambda+1)^{2} p}{\lambda p+1} \text { and } \psi_{a}(z)=-\ln (1-a)+\ln (a) \sum_{n=0}^{\infty} \frac{a^{n+z}}{1-a^{n+z}} \text {, being } \psi_{a}(z)
\end{gathered}
$$

known as the a-digama function.

(ii) If $p=\left(\lambda^{2}+\lambda+1\right)^{-1}$ then

$$
\mathbb{P}(M \leq m)=\frac{(m+1) \lambda}{(m+1) \lambda+1}
$$

and

$$
\mathbb{E}(M)=\infty
$$

\subsection{Number of collonies in the process.}

Theorem 3.20. Consider the process $\left\{\mathbb{T}^{d} ; N\right\}$. If

$$
\mathbb{E}\left[\left(\frac{d}{d+1}\right)^{N}\right]>\frac{d-1}{d}
$$

then

$$
\begin{gathered}
\mathbb{E}\left(I_{d}\right) \leq(1-\beta)^{-1} \text { and } \\
\mathbb{E}\left(I_{d}\right) \geq \sum_{r=1}^{d+1}\left[[1+r \theta]\left(\begin{array}{c}
d+1 \\
r
\end{array}\right) \sum_{n=r}^{\infty} \frac{T(n, r)}{(d+1)^{n}} \mathbb{P}(N=n)\right]+\mathbb{P}(N=0)
\end{gathered}
$$

where $\theta=(1-\alpha)^{-1}$. Besides that, if $\mathbb{E}(N)<1$ (the subcritical case)

$$
\lim _{d \rightarrow \infty} \mathbb{E}\left(I_{d}\right)=\frac{1}{1-\mathbb{E}(N)} .
$$

\section{ProOfs}

In order to prove the main results we define auxiliary processes whose understanding will provide bounds for the processes defined at introduction. In the first two auxiliary process, denoted by $U\left\{\mathbb{T}^{d} ; N\right\}$ and $U\left\{\mathbb{T}_{+}^{d} ; N\right\}$, every time a colony collapses the survival individuals are only allowed to choose neighbour vertices which are further (compared to the origin) that the vertex where their colony was placed. In other words an individual is not allowed to choose the neighbour vertex which has been already colonized. We refer 
to this process as Self Avoiding. The last two auxiliary process, denoted by $L\left\{\mathbb{T}^{d} ; N\right\}$ and $L\left\{\mathbb{T}_{+}^{d} ; N\right\}$, while the survival individuals are allowed to choose the neighbour vertex which has been already colonized, those who does that are not able to colonize it as this place is considered hostile or infertile. We refer to this process as Move Forward or Die. In both processes, $Y$, the number of new colonies at collapse times in a vertex $x$ equals the number of diferent neighbours chosen which are located further from the origin than $x$ is. Besides that, every new colony starts with only one individual.

Proposition 4.1. Consider a sequence of random variables $\left\{Y_{d}\right\}_{d \in \mathbb{N}}$ whose sequence of probability generating functions is $\left\{g_{Y_{d}}(s)\right\}_{d \in \mathbb{N}}$ and a random variable $Y$ such that $Y_{d} \stackrel{D}{\rightarrow} Y$. Then $g_{Y_{d}, m}(s)$, the $m$ - th composition of $g_{Y_{d}}(s)$, converges to $g_{Y, m}(s)$, where $g_{Y, m}(s)$ is the $m-$ th composition of $g_{Y}(s)$, the probability generating function of $Y$.

Proof of Proposition 4.1. From the fact that $Y_{d} \stackrel{D}{\rightarrow} Y$ it follows that $g_{Y}(s)=\lim _{d \rightarrow \infty} g_{Y_{d}}(s)$.

$$
\lim _{d \rightarrow \infty} g_{Y_{d}, 2}(s)=\lim _{d \rightarrow \infty} g_{Y_{d}}\left(g_{Y_{d}}(s)\right)=\lim _{d \rightarrow \infty} \mathbb{E}\left[\left(\mathbb{E}\left(s^{Y_{d}}\right)\right)^{Y_{d}}\right]
$$

From the Dominated Convergence Theorem [14, Theorem 9.1 page 26] (observe that $\left.\left[\mathbb{E}\left(s^{Y_{d}}\right)\right]^{Y_{d}} \in[0,1]\right)$

$$
\lim _{d \rightarrow \infty} \mathbb{E}\left[\left[\mathbb{E}\left(s^{Y_{d}}\right)\right]^{Y_{d}}\right]=\mathbb{E}\left[\lim _{d \rightarrow \infty}\left[\mathbb{E}\left(s^{Y_{d}}\right)\right]^{Y_{d}}\right] .
$$

Again, from the Dominated Convergence Theorem [14, Theorem 9.1 page 26] (observe that $s^{Y_{d}} \in[0,1]$ and that $Y_{d}$ converges to $Y$ in distribution) $Y_{d} \ln \mathbb{E}\left(s^{Y_{d}}\right)$ converges in distribution to $Y \ln \mathbb{E}\left(s^{Y}\right)$. So we conclude that

$$
\mathbb{E}\left[\lim _{d \rightarrow \infty}\left(\mathbb{E}\left(s^{Y_{d}}\right)\right)^{Y_{d}}\right]=\mathbb{E}\left[\left[\mathbb{E}\left(s^{Y}\right)\right]^{Y}\right]
$$

and then

$$
\lim _{d \rightarrow \infty} g_{Y_{d}, 2}(s)=\lim _{d \rightarrow \infty} \mathbb{E}\left[\left(\mathbb{E}\left(s^{Y_{d}}\right)\right)^{Y_{d}}\right]=\mathbb{E}\left[\left[\mathbb{E}\left(s^{Y}\right)\right]^{Y}\right]=g_{Y, 2}(s) .
$$

By induction one can prove that $\lim _{d \rightarrow \infty} g_{Y_{d}, m}(s)=g_{Y, m}(s)$.

Proposition 4.2. Let $\left\{Z_{n}\right\}_{n \geq 0},\left\{Z_{1, n}\right\}_{n \geq 0},\left\{Z_{2, n}\right\}_{n \geq 0}, \cdots$ be a branching processes and $Y, Y_{1}, Y_{2}, \cdots$, respectively, their offspring distributions. Supose that

(i) $Y_{d} \stackrel{D}{\rightarrow} Y$;

(ii) $\mathbb{P}\left(Y_{d} \geq k\right) \leq \mathbb{P}\left(Y_{d+1} \geq k\right)$, for all $k$ and for all $d$;

Then, if $\nu_{d}$ is the probability of the extinction of the process $\left\{Z_{d, n}\right\}_{n \geq 0}$ and $\nu$ is the probability of the extinction of the process $\left\{Z_{n}\right\}_{n \geq 0}$ we have that

$$
\lim _{d \rightarrow \infty} \nu_{d}=\nu
$$

Proof of Proposition 4.2. From (i), (ii) and by using a coupling argument we have that

$$
\nu_{d} \geq \nu_{d+1} \geq \lim _{d \rightarrow \infty} \nu_{d}=: \nu_{L} \geq \nu
$$

From the fact that $Y_{d} \stackrel{D}{\rightarrow} Y$ and [4, Theorem 25.8, page 335] we have that 


$$
\phi_{d}(s):=\mathbb{E}\left[s^{Y_{d}}\right] \underset{d \rightarrow \infty}{\longrightarrow} \mathbb{E}\left[s^{Y}\right]:=\phi(s) .
$$

Let $s \in[0,1]$ fixed and $f(y):=s^{y}, y \in \mathbb{N}$. Clearly, $f$ is non-increasing and therefore from (ii) and [14, equation (3.3), page 6] we have that

$$
\phi_{d+1}(s) \leq \phi_{d}(s) \text {. }
$$

From 4.2), 4.3) and Dini's Theorem, we have that

$$
\phi_{d}(\cdot) \longrightarrow \phi(\cdot) \text { uniformly. }
$$

From 4.1], 4.4 and [13, Exercise 9 - Chapter 7]:

$$
\lim _{d \rightarrow \infty} \phi_{d}\left(\nu_{d}\right)=\phi\left(\nu_{L}\right)
$$

Finally, given that $\phi_{d}\left(\nu_{d}\right)=\nu_{d}$, from 4.5 we obtain that

$$
\phi\left(\nu_{L}\right)=\nu_{L}
$$

From the convexity of $\phi(s)$ it follows that $\phi(s)=s$ (the fixed points of $\phi(\cdot)$ ) for at most two points in $[0,1]$. It is known that [see [6], Theorem 6.1 and its proof] if $\nu<1$, the fixed points of $\phi(\cdot)$ are $s=\nu$ and $s=1$. If $\nu=1$, the unique solution is 1 . So there are two cases to be considered.

1. If $\nu_{d}<1$ for some $d \geq 1$, then from (4.1) it follows that $\nu_{L}<1$. If $\nu_{L}<1$, it follows from (4.6) that $\nu_{L}=\nu$.

2. If $\nu_{d}=1$ for all $d \geq 1$, then

$$
\mathbb{E}\left(Y_{d}\right) \leq 1 \text { for all } d \geq 1
$$

Then,

$$
\lim _{d \rightarrow \infty} \mathbb{E}\left(Y_{d}\right) \leq 1
$$

From (ii) we have that $\mathbb{P}\left(Y_{d} \geq k\right) \leq \mathbb{P}(Y \geq k)$, for all $k$ and all $d$. From (i), (ii) and a non standart version of Fatou Lemma [2, page 230] (applied to the sequence $a_{d, j}=$ $\left.j \mathbb{P}\left(Y_{d}=j\right)\right)$, it follows that

$$
\liminf _{d \rightarrow \infty} \mathbb{E}\left(Y_{d}\right) \geq \mathbb{E}(Y) .
$$

From (4.7) and (4.8), it follows that $\nu=1$. Then, from (4.6) we have that $\nu_{L}=\nu$.

\section{1. $U\left\{\mathbb{T}^{d} ; N\right\}$ : The Self Avoiding model.}

Proposition 4.3. Consider the process $U\left\{\mathbb{T}^{d} ; N\right\} . \mathbb{P}\left(V_{d}\right)>0$ if and only if

$$
\mathbb{E}\left[\left(\frac{d-1}{d}\right)^{N}\right]<\frac{d-1}{d}
$$

Proof of Proposition 4.3. First of all observe that for a fixed distribution for $N$, the processes $U\left\{\mathbb{T}^{d} ; N\right\}$ and $U\left\{\mathbb{T}_{+}^{d} ; N\right\}$ either both survives or both die. Next observe that the process $U\left\{\mathbb{T}_{+}^{d} ; N\right\}$ behaves as a homogeneous branching process. Every vertex $x$ which is colonized produces $Y_{d}$ new colonies (whose distribution depends only on $N$ ) on the $d$ 
neighbour vertices which located are further from the origin than $x$ is. By conditioning one can see that

$$
\mathbb{E}\left(Y_{d}\right)=d \sum_{n=0}^{\infty}\left[\left(1-\left(\frac{d-1}{d}\right)^{n}\right) \mathbb{P}(N=n)\right]=d\left[1-\mathbb{E}\left[\left(\frac{d-1}{d}\right)^{N}\right]\right] .
$$

From the theory of homogeneous branching processes we see that $U\left\{\mathbb{T}_{+}^{d} ; N\right\}$ (and also $\left.U\left\{\mathbb{T}^{d} ; N\right\}\right)$ survives if and only if $\mathbb{E}\left[\left(\frac{d-1}{d}\right)^{N}\right]<\frac{d-1}{d}$.

Proposition 4.4. Consider the process $U\left\{\mathbb{T}^{d} ; N\right\}$. Then

$$
\mathbb{P}\left(V_{d}\right)=\sum_{r=1}^{d+1}\left[\left(1-\psi^{r}\right)\left(\begin{array}{c}
d+1 \\
r
\end{array}\right) \sum_{n=r}^{\infty} \frac{T(n, r)}{(d+1)^{n}} \mathbb{P}(N=n)\right]
$$

where $\psi$, the extinction probability for the process $U\left\{\mathbb{T}_{+}^{d} ; N\right\}$, is the smallest non-negative solution of

$$
\sum_{y=0}^{d}\left[s^{y}\left(\begin{array}{l}
d \\
y
\end{array}\right) \sum_{n=y}^{\infty} \frac{T(n, y)}{d^{n}} \mathbb{P}(N=n)\right]=s .
$$

On the sub critical regime, which means

$$
\mathbb{E}\left[\left(\frac{d-1}{d}\right)^{N}\right]>\frac{d-1}{d}
$$

it holds that

$$
\mathbb{E}\left(I_{d}\right)=\sum_{r=1}^{d}\left[\left[1+r \theta_{u}\right]\left(\begin{array}{c}
d+1 \\
r
\end{array}\right) \sum_{n=r}^{\infty} \frac{T(n, r)}{(d+1)^{n}} \mathbb{P}(N=n)\right]+\mathbb{P}(N=0)
$$

where

$$
\theta_{u}=\left\{1-d\left[1-\mathbb{E}\left(\left(\frac{d-1}{d}\right)^{N}\right)\right]\right\}^{-1} .
$$

Proof of Proposition 4.4. Let $Y_{d, R}$ be the number of colonies created at the neighbour vertices of the origin from its colony at the collapse time. Then

$$
\mathbb{P}\left(V_{d}\right)=\sum_{r=0}^{d+1} \mathbb{P}\left(V_{d} \mid Y_{d, R}=r\right) \mathbb{P}\left(Y_{d, R}=r\right)
$$

where

$$
\mathbb{P}\left(Y_{d, R}=r\right)=\sum_{n=r}^{\infty}\left[\mathbb{P}(N=n) \frac{\left(\begin{array}{c}
d+1 \\
r
\end{array}\right) T(n, r)}{(d+1)^{n}}\right] \text { for } r=0,1,2, \cdots, d+1
$$


because

$$
\mathbb{P}\left(Y_{d, R}=r \mid N=n\right)=\frac{\left(\begin{array}{c}
d+1 \\
r
\end{array}\right) T(n, r)}{(d+1)^{n}} .
$$

Given that $Y_{d, R}=r$ one have $r$ independent $U\left\{\mathbb{T}_{+}^{d} ; N\right\}$ processes living on $r$ independent rooted trees. Every vertex $x$ which is colonized, on some of these trees, right after the collapse will have $N$ survival individuals. These individuals will produce $Y_{d}$ new colonies (whose distribution depends only on $N$ ) on the $d$ neighbour vertices which are located further from the origin than $x$ is. So we have that

$$
\mathbb{P}\left(Y_{d}=y \mid N=n\right)=\frac{\left(\begin{array}{l}
d \\
y
\end{array}\right) T(n, y)}{d^{n}} .
$$

From this,

$$
\mathbb{P}\left(Y_{d}=y\right)=\sum_{n=y}^{\infty}\left[\mathbb{P}(N=n) \frac{\left(\begin{array}{l}
d \\
y
\end{array}\right) T(n, y)}{d^{n}}\right] \text { for } y=0,1,2, \cdots, d
$$

and

$$
\mathbb{E}\left(s^{Y_{d}}\right)=\sum_{y=0}^{d}\left[s^{y}\left(\begin{array}{l}
d \\
y
\end{array}\right) \sum_{n=y}^{\infty} \frac{T(n, y)}{d^{n}} \mathbb{P}(N=n)\right] .
$$

Then $\mathbb{P}\left(V_{d}^{C} \mid Y_{d, R}=r\right)=\psi^{r}$ for $r=0,1,2, \cdots, d+1$ and

$$
\mathbb{P}\left(V_{d}\right)=\sum_{r=1}^{d+1}\left[\left(1-\psi^{r}\right)\left(\begin{array}{c}
d+1 \\
r
\end{array}\right) \sum_{n=r}^{\infty} \frac{T(n, r)}{(d+1)^{n}} \mathbb{P}(N=n)\right]
$$

As for the second part of the proposition

$$
\mathbb{E}\left(I_{d}\right)=\sum_{r=0}^{d+1} \mathbb{E}\left(I_{d} \mid Y_{d, R}=r\right) \mathbb{P}\left(Y_{d, R}=r\right) .
$$

Besides that, $\mathbb{E}\left(I_{d} \mid Y_{d, R}=r\right)=r \theta_{u}+1$ (see Stirzaker [9, Exercise 2b, page 280]).

Proposition 4.5. Consider the process $U\left\{\mathbb{T}^{d} ; N\right\}$. Then

$$
\lim _{d \rightarrow \infty} \mathbb{P}\left(V_{d}\right)=1-\nu
$$

where $\nu$ is the smallest non-negative solution of $\mathbb{E}\left(s^{N}\right)=s$. Besides that, if $\mathbb{E}(N)<1$ (the subcritical case) then

$$
\lim _{d \rightarrow \infty} \mathbb{E}\left(I_{d}\right)=\frac{1}{1-\mathbb{E}(N)}
$$


Proof of Proposition 4.5. In order to prove 4.10 one has to apply Proposition 4.2, observing that $Y_{d} \stackrel{D}{\rightarrow} N$ and $Y_{d, R} \stackrel{D}{\rightarrow} N$. Moreover to prove 4.11 observe that

$$
\lim _{d \rightarrow \infty} \mathbb{E}\left(I_{d}\right)=\lim _{d \rightarrow \infty} \sum_{r=0}^{d+1} \mathbb{E}\left(I_{d} \mid Y_{d, R}=r\right) \mathbb{P}\left(Y_{d, R}=r\right) .
$$

As $Y_{d} \stackrel{D}{\rightarrow} N$ and $Y_{d, R} \stackrel{D}{\rightarrow} N$ then

$$
\lim _{d \rightarrow \infty} \mathbb{E}\left(I_{d} \mid Y_{d, R}=r\right)=\lim _{d \rightarrow \infty} r \theta_{u}+1=\frac{r}{1-\mathbb{E}(N)}+1
$$

and the result follows from the Dominated Convergence Theorem [14, Theorem 9.1 page 26].

Proposition 4.6. Consider the process $U\left\{\mathbb{T}_{+}^{d} ; N\right\}$. Assuming

$$
\mathbb{E}\left[\left(\frac{d-1}{d}\right)^{N}\right]>\frac{d-1}{d}
$$

we have that

$$
\frac{[1+D(1-\mu)]\left[1-\mu^{m+1}\right]}{1+D(1-\mu)-\mu^{m+1}} \leq \mathbb{P}\left(M_{d} \leq m\right) \leq \frac{\left[1+\frac{\mu(1-\mu)}{B}\right]\left(1-\mu^{m+1}\right)}{1+\frac{\mu(1-\mu)}{B}-\mu^{m+1}}
$$

and

$$
\frac{\mu^{2}}{2(B+\mu)}+\mu(1-\mu) \frac{\ln \left[1-\frac{\mu B}{B+\mu(1-\mu)}\right]}{B \ln \mu} \leq \mathbb{E}\left(M_{d}\right) \leq \frac{D \mu}{D+1}+D(1-\mu) \frac{\ln \left[1-\frac{\mu}{1+D(1-\mu)}\right]}{\ln \mu}
$$

where

$$
\begin{aligned}
& \mu=d\left[1-\mathbb{E}\left[\left(\frac{d}{d+1}\right)^{N}\right]\right] \\
& D=\max \left\{2 ; \frac{g^{\prime}(1)}{g^{\prime}(1)-\mathbb{P}(N \neq 0)}\right\} \\
& B=d(d-1)\left[1-2 \mathbb{E}\left(\left(\frac{d-1}{d}\right)^{N}\right)+\mathbb{E}\left(\left(\frac{d-2}{d}\right)^{N}\right)\right] .
\end{aligned}
$$

Moreover,

$$
M_{d} \stackrel{D}{\rightarrow} M
$$

where $\mathbb{P}(M \leq m)=g_{m+1}(0)$, being $g(s)=\mathbb{E}\left(s^{N}\right)$ and $g_{m+1}(s)=g(g(\cdots g(s)) \cdots)$.

Proof of Proposition 4.6. Every vertex $x$ which is colonized produces $Y_{d}$ new colonies (whose distribution depends only on $N$ ) on the $d$ neighbour vertices which are located further from the origin than $x$ is. The random variable $Y_{d}$ can be seen as $Y_{d}=\sum_{i=1}^{d} I_{i}$ 
where for $i=1, \ldots, d$

$$
I_{i}= \begin{cases}1, & \text { the } i-t h \text { neighbour of } x \text { is colonized } \\ 0, & \text { else }\end{cases}
$$

Defining $g_{Y_{d}}(s)$ as the generating function of $Y_{d}$ observe that equation 4.9 gives $g_{Y_{d}}^{\prime}(1)$. Moreover

$$
Y_{d}^{2}=\left(\sum_{i=1}^{d} I_{i}\right)^{2}=\sum_{i=1}^{d} I_{i}^{2}+2 \sum_{1 \leq i<j \leq d} I_{i} I_{j}
$$

and

$$
\mathbb{E}\left(Y_{d}^{2}\right)=d \mathbb{E}\left(I_{1}^{2}\right)+d(d-1) \mathbb{E}\left(I_{1} I_{2}\right)
$$

and finally

$$
\mathbb{E}\left(Y_{d}^{2}\right)=d\left[1-\mathbb{E}\left[\left(\frac{d-1}{d}\right)^{N}\right]\right]+d(d-1)\left[1-2 \mathbb{E}\left[\left(\frac{d-1}{d}\right)^{N}\right]+\mathbb{E}\left[\left(\frac{d-2}{d}\right)^{N}\right]\right]
$$

Then

$$
g_{Y_{d}}^{\prime \prime}(1)=\mathbb{E}\left(Y_{d}\left(Y_{d}-1\right)\right)=d(d-1)\left[1-2 \mathbb{E}\left[\left(\frac{d-1}{d}\right)^{N}\right]+\mathbb{E}\left[\left(\frac{d-2}{d}\right)^{N}\right]\right] .
$$

Then the result follows from Theorem 1 page 331 in [3], where $m=g_{Y_{d}}^{\prime}(1)$.

The convergence $M_{d} \stackrel{D}{\rightarrow} M$ follows from the fact that $Y_{d} \stackrel{D}{\rightarrow} N$ when $d \rightarrow \infty$ and from Proposition 4.1.

\section{2. $L\left\{\mathbb{T}^{d} ; N\right\}$ : Move Forward or Die.}

Proposition 4.7. Consider the process $L\left\{\mathbb{T}^{d} ; N\right\} . \mathbb{P}\left(V_{d}\right)>0$ if and only if

$$
\mathbb{E}\left[\left(\frac{d}{d+1}\right)^{N}\right]<\frac{d-1}{d}
$$

Proof of Proposition 4.7. First of all observe that for a fixed distribution for $N$, the processes $L\left\{\mathbb{T}^{d} ; N\right\}$ and $L\left\{\mathbb{T}_{+}^{d} ; N\right\}$ either both survives or both die. Next observe that the process $L\left\{\mathbb{T}_{+}^{d} ; N\right\}$ behaves as a homogeneous branching process. Every vertex $x$ which is colonized produces a bunch of survival individuals right after the collapse which are willing to jump to one of the $d+1$ nearest neighbours vertices of $x$. All those which jump towards the origin get killed. So, $Y_{d}$ new colonies will be found on the $d$ neighbour vertices which are located further from the origin than $x$ is. By conditioning one can see that

$$
\mathbb{E}\left(Y_{d}\right)=d \sum_{n=0}^{\infty}\left[\left(1-\left(\frac{d}{d+1}\right)^{n}\right) \mathbb{P}(N=n)\right]=d\left[1-\mathbb{E}\left[\left(\frac{d}{d+1}\right)^{N}\right]\right]
$$

From the theory of homogeneous branching processes we see that $L\left\{\mathbb{T}_{+}^{d} ; N\right\}$ (and also $\left.L\left\{\mathbb{T}^{d} ; N\right\}\right)$ survives if and only if $\mathbb{E}\left[\left(\frac{d}{d+1}\right)^{N}\right]<\frac{d-1}{d}$. 
Proposition 4.8. Consider the process $L\left\{\mathbb{T}^{d} ; N\right\}$. Then

$$
\mathbb{P}\left(V_{d}\right)=\sum_{r=1}^{d+1}\left[\left(1-\rho^{r}\right)\left(\begin{array}{c}
d+1 \\
r
\end{array}\right) \sum_{n=r}^{\infty} \frac{T(n, r)}{(d+1)^{n}} \mathbb{P}(N=n)\right]
$$

where $\rho$, the extinction probability for the process $L\left\{\mathbb{T}_{+}^{d} ; N\right\}$, is the smallest non-negative solution of

$$
\sum_{y=0}^{d}\left[s^{y}\left(\begin{array}{l}
d \\
y
\end{array}\right) \sum_{n=y}^{\infty} \frac{T(n, y)+T(n, y+1)}{(d+1)^{n}} \mathbb{P}(N=n)\right]=s .
$$

On the subcritical regime, which means

$$
\mathbb{E}\left[\left(\frac{d}{d+1}\right)^{N}\right]>\frac{d-1}{d}
$$

it holds that

$$
\mathbb{E}\left(I_{d}\right)=\sum_{r=1}^{d+1}\left[\left[1+r \theta_{l}\right]\left(\begin{array}{c}
d+1 \\
r
\end{array}\right) \sum_{n=r}^{\infty} \frac{T(n, r)}{(d+1)^{n}} \mathbb{P}(N=n)\right]+\mathbb{P}(N=0)
$$

where

$$
\theta_{l}=\left\{1-d\left[1-\mathbb{E}\left(\left(\frac{d}{d+1}\right)^{N}\right)\right]\right\}^{-1}
$$

Proof of Proposition 4.8. Let $Y_{d, R}$ be the number of colonies created at the neighbour vertices of the origin from its colony at the collapse time. Then

$$
\mathbb{P}\left(V_{d}\right)=\sum_{r=0}^{d+1} \mathbb{P}\left(V_{d} \mid Y_{d, R}=r\right) \mathbb{P}\left(Y_{d, R}=r\right)
$$

where

$$
\mathbb{P}\left(Y_{d, R}=r\right)=\sum_{n=r}^{\infty}\left[\mathbb{P}(N=n) \frac{\left(\begin{array}{c}
d+1 \\
r
\end{array}\right) T(n, r)}{(d+1)^{n}}\right] \text { for } r=0,1,2, \cdots, d+1
$$

because

$$
\mathbb{P}\left(Y_{d, R}=r \mid N=n\right)=\frac{\left(\begin{array}{c}
d+1 \\
r
\end{array}\right) T(n, r)}{(d+1)^{n}} .
$$

Given that $Y_{d, R}=r$ one have $r$ independent $L\left\{\mathbb{T}_{+}^{d} ; N\right\}$ processes living on $r$ independent rooted trees. Every vertex $x$ which is colonized, on some of these trees, right after the collapse will have $N$ survival individuals. These individuals will produce $Y_{d}$ new colonies (whose distribution depends only on $N$ ) on the $d$ neighbour vertices which are located further from the origin than $x$ is. So we have that 
From this,

$$
\mathbb{P}\left(Y_{d}=y \mid N=n\right)=\frac{\left(\begin{array}{l}
d \\
y
\end{array}\right)[T(n, y)+T(n, y+1)]}{(d+1)^{n}}
$$

$$
\mathbb{P}\left(Y_{d}=y\right)=\sum_{n=y}^{\infty}\left[\mathbb{P}(N=n) \frac{\left(\begin{array}{l}
d \\
y
\end{array}\right)[T(n, y)+T(n, y+1)]}{(d+1)^{n}}\right] \text { for } y=0,1,2, \cdots, d
$$

and

$$
\mathbb{E}\left(s^{Y_{d}}\right)=\sum_{y=0}^{d} s^{y} \sum_{n=y}^{\infty}\left[\mathbb{P}(N=n) \frac{\left(\begin{array}{l}
d \\
y
\end{array}\right)[T(n, y)+T(n, y+1)]}{(d+1)^{n}}\right]
$$

Then $\mathbb{P}\left(V_{d}^{C} \mid Y_{d, R}=r\right)=\rho^{r}, r=0,1,2, \cdots, d+1$ and

$$
\mathbb{P}\left(V_{d}\right)=\sum_{r=1}^{d+1}\left[\left(1-\rho^{r}\right)\left(\begin{array}{c}
d+1 \\
r
\end{array}\right) \sum_{n=r}^{\infty} \frac{T(n, r)}{(d+1)^{n}} \mathbb{P}(N=n)\right]
$$

As for the second part of the proposition

$$
\mathbb{E}\left(I_{d}\right)=\sum_{r=0}^{d+1} \mathbb{E}\left(I_{d} \mid Y_{d, R}=r\right) \mathbb{P}\left(Y_{d, R}=r\right) .
$$

Besides that, $\mathbb{E}\left(I_{d} \mid Y_{d, R}=r\right)=r \theta_{l}+1$ (see Stirzaker [9, Exercise 2b, page 280]).

Proposition 4.9. Consider the process $L\left\{\mathbb{T}^{d} ; N\right\}$. Then,

$$
\lim _{d \rightarrow \infty} \mathbb{P}\left(V_{d}\right)=1-\nu
$$

where $\nu$ is the smallest non-negative solution of $\mathbb{E}\left(s^{N}\right)=s$. Besides that, if $\mathbb{E}(N)<1$ (the subcritical case) then

$$
\lim _{d \rightarrow \infty} \mathbb{E}\left(I_{d}\right)=\frac{1}{1-\mathbb{E}(N)}
$$

Proof of Proposition 4.9. In order to prove 4.13 one has to aply Proposition 4.2, observing that $Y_{d} \stackrel{D}{\rightarrow} N$ and $Y_{d, R} \stackrel{D}{\rightarrow} N$. For the proof of 4.14 observe that

$$
\lim _{d \rightarrow \infty} \mathbb{E}\left(I_{d}\right)=\lim _{d \rightarrow \infty} \sum_{r=0}^{d+1} \mathbb{E}\left(I_{d} \mid Y_{d, R}=r\right) \mathbb{P}\left(Y_{d, R}=r\right) .
$$

As $Y_{d} \stackrel{D}{\rightarrow} N$ and $Y_{d, R} \stackrel{D}{\rightarrow} N$ then

$$
\lim _{d \rightarrow \infty} \mathbb{E}\left(I_{d} \mid Y_{d, R}=r\right)=\lim _{d \rightarrow \infty} r \theta_{l}+1=\frac{r}{1-\mathbb{E}(N)}+1 .
$$


The result follows from the Dominated Convergence Theorem [14, Theorem 9.1 page 26].

Proposition 4.10. Consider the process $L\left\{\mathbb{T}_{+}^{d} ; N\right\}$. Assuming

$$
\mathbb{E}\left[\left(\frac{d}{d+1}\right)^{N}\right]>\frac{d-1}{d}
$$

We have that

$$
\frac{[1+D(1-\mu)]\left[1-\mu^{m+1}\right]}{1+D(1-\mu)-\mu^{m+1}} \leq \mathbb{P}\left(M_{d} \leq m\right) \leq \frac{\left[1+\frac{\mu(1-\mu)}{B}\right]\left(1-\mu^{m+1}\right)}{1+\frac{\mu(1-\mu)}{B}-\mu^{m+1}}
$$

and

$$
\frac{\mu^{2}}{2(B+\mu)}+\mu(1-\mu) \frac{\ln \left[1-\frac{\mu B}{B+\mu(1-\mu)}\right]}{B \ln \mu} \leq \mathbb{E}\left(M_{d}\right) \leq \frac{D \mu}{D+1}+D(1-\mu) \frac{\ln \left[1-\frac{\mu}{1+D(1-\mu)}\right]}{\ln \mu}
$$

where

$$
\begin{aligned}
& \mu=d\left[1-\mathbb{E}\left[\left(\frac{d}{d+1}\right)^{N}\right]\right] \\
& D=\max \left\{2 ; \frac{\mu}{\mu-\mathbb{P}(N \neq 0)}\right\} \\
& B=d(d-1)\left[1-2 \mathbb{E}\left(\left(\frac{d}{d+1}\right)^{N}\right)+\mathbb{E}\left(\left(\frac{d-1}{d+1}\right)^{N}\right)\right] .
\end{aligned}
$$

Besides that,

$$
M_{d} \stackrel{D}{\rightarrow} M
$$

where $\mathbb{P}(M \leq m)=g_{m+1}(0)$, being $g(s)=\mathbb{E}\left(s^{N}\right)$ and $g_{m+1}(s)=g\left(g\left(\begin{array}{c}m+1 \text { times } \\ \cdots\end{array}(s)\right) \cdots\right)$.

Proof of Proposition 4.10. Every vertex $x$ which is colonized produces $Y_{d}$ new colonies (whose distribution depends only on $N$ ) on the $d$ neighbour vertices which are located further from the origin than $x$ is. The random variable $Y_{d}$ can be seen as $Y_{d}=\sum_{i=1}^{d} I_{i}$ where for $i=1, \ldots, d$

$$
I_{i}= \begin{cases}1, & \text { the } i-t h \text { neighbour of } x \text { is colonized } \\ 0, & \text { else. }\end{cases}
$$

Defining $g_{Y_{d}}(s)$ as the generating function of $Y_{d}$ observe that equation 4.12 gives $g_{Y_{d}}^{\prime}(1)$. Moreover

$$
Y_{d}^{2}=\left(\sum_{i=1}^{d} I_{i}\right)^{2}=\sum_{i=1}^{d} I_{i}^{2}+2 \sum_{1 \leq i<j \leq d} I_{i} I_{j}
$$

and

$$
\mathbb{E}\left(Y_{d}^{2}\right)=d \mathbb{E}\left(I_{1}^{2}\right)+d(d-1) \mathbb{E}\left(I_{1} I_{2}\right)
$$


and finally

$$
\mathbb{E}\left(Y_{d}^{2}\right)=d\left[1-\mathbb{E}\left[\left(\frac{d}{d+1}\right)^{N}\right]\right]+d(d-1)\left[1-2 \mathbb{E}\left[\left(\frac{d}{d+1}\right)^{N}\right]+\mathbb{E}\left[\left(\frac{d-1}{d+1}\right)^{N}\right]\right]
$$

Then

$$
g_{Y_{d}}^{\prime \prime}(1)=\mathbb{E}\left(Y_{d}\left(Y_{d}-1\right)\right)=d(d-1)\left[1-2 \mathbb{E}\left[\left(\frac{d}{d+1}\right)^{N}\right]+\mathbb{E}\left[\left(\frac{d-1}{d+1}\right)^{N}\right]\right]
$$

Then the result follows from Theorem 1 page 331 in [3], where $m=g_{Y_{d}}^{\prime}(1)$.

The convergence $M_{d} \stackrel{D}{\rightarrow} M$ follows from the fact that $Y_{d} \stackrel{D}{\rightarrow} N$ when $d \rightarrow \infty$ and from Proposition 4.1 .

\subsection{Proofs of the main results.}

First we define a coupling between the processes $\left\{\mathbb{T}^{d} ; N\right\}$ and $L\left\{\mathbb{T}_{+}^{d} ; N\right\}$ in such a way that the former is dominated by the earlier. Every colony in $L\left\{\mathbb{T}_{+}^{d} ; N\right\}$ is associated to a colony in $\left\{\mathbb{T}^{d} ; N\right\}$. As a consequence, if the process $\left\{\mathbb{T}^{d} ; N\right\}$ dies out, the same happens to $L\left\{\mathbb{T}_{+}^{d} ; N\right\}$.

At every collapse time at a vertex $x$ in the original model, a non-empty group of individuals that tries to colonize the neighbour vertex to $x$ which is closer to the origin than $x$ will create there a new colony provided that that vertex is empty. In the model $L\left\{\mathbb{T}_{+}^{d} ; N\right\}$ the same non-empty group of individuals that tries to colonize the same vertex, imediately dies.

Next we define a coupling between the processes $\left\{\mathbb{T}^{d} ; N\right\}$ and $U\left\{\mathbb{T}_{+}^{d+1} ; N\right\}$ in such a way that the former dominates the earlier. Every colony in $\left\{\mathbb{T}^{d} ; N\right\}$ can be associated to a colony in $U\left\{\mathbb{T}_{+}^{d+1} ; N\right\}$. As a consequence if the process $U\left\{\mathbb{T}_{+}^{d+1} ; N\right\}$ dies out, the same happens to $\left\{\mathbb{T}^{d} ; N\right\}$.

At every collapse time at a vertex $x$ we associate the neighbour vertex to $x$ which is closer to the origin than $x$ to the extra vertex on the model $U\left\{\mathbb{T}_{+}^{d+1} ; N\right\}$. In the original model, a non-empty group of individuals that tries to colonize the neighbour vertex to $x$ which is closer to the origin than $x$ will create there a new colony provided that that vertex is empty. In the model $U\left\{\mathbb{T}_{+}^{d+1} ; N\right\}$ the same non-empty group of individuals that tries to colonize the extra vertex, founds a new colonony there.

Proof of Theorem 3.2. The result follows from the fact that the process $\left\{\mathbb{T}^{d} ; N\right\}$ dominates the process $L\left\{\mathbb{T}_{+}^{d} ; N\right\}$ and by its turn, is dominated by the process $U\left\{\mathbb{T}_{+}^{d+1} ; N\right\}$, together with Propositions 4.3 and 4.7 .

Proof of Corollary 3.3. Assuming $s=\frac{d}{d+1}$ in 2.1 and applying Theorem 3.2 the result follows.

Proof of Corollary 3.4. Assuming $s=\frac{d}{d+1}$ in 2.3 and applying Theorem 3.2 the result follows. 
Proof of Theorem 3.9. The result follows from the fact that the process $\left\{\mathbb{T}^{d} ; N\right\}$ dominates the process $L\left\{\mathbb{T}_{+}^{d} ; N\right\}$ and by its turn, is dominated by the process $U\left\{\mathbb{T}_{+}^{d+1} ; N\right\}$, together with Propositions 4.4 and 4.8 .

Proof of Theorem 3.10. The result follows from the fact that the process $\left\{\mathbb{T}^{d} ; N\right\}$ dominates the process $L\left\{\mathbb{T}_{+}^{d} ; N\right\}$ and by its turn, is dominated by the process $U\left\{\mathbb{T}_{+}^{d+1} ; N\right\}$, together with Propositions 4.5 and 4.9 .

Proof of Corollary 3.11. The proof is just a matter of computing the smallest positive fixed point for the generating function of $N$ (the smallest positive $s$ such that $\mathbb{E}\left(s^{N}\right)=s$ ) for $\mathbb{E}\left(s^{N}\right)$ given in 2.1 .

Proof of Corollary 3.13. The proof is just a matter of computing the smallest positive fixed point for the generating function of $N$ (the smallest positive $s$ such that $\mathbb{E}\left(s^{N}\right)=s$ ) for $\mathbb{E}\left(s^{N}\right)$ given in 2.3 .

Proof of Theorem 3.16. The result follows from the fact that the process $\left\{\mathbb{T}^{d} ; N\right\}$ dominates the process $L\left\{\mathbb{T}_{+}^{d} ; N\right\}$ and by its turn, is dominated by the process $U\left\{\mathbb{T}_{+}^{d+1} ; N\right\}$, together with Propositions 4.6 and 4.10 .

Proof of Corollary 3.17. The proof is just a matter of computing the generating function of $N$ (see Equation (2.1)) on both values $s=\frac{d}{d+1}$ and $s=\frac{d-1}{d+1}$.

Proof of Theorem 3.18. The result follows from the fact that the process $\left\{\mathbb{T}^{d} ; N\right\}$ dominates the process $L\left\{\mathbb{T}_{+}^{d} ; N\right\}$ and by its turn, is dominated by the process $U\left\{\mathbb{T}_{+}^{d+1} ; N\right\}$, together with Propositions 4.6 and 4.10

Definition 4.11. A fractional linear generating function is a probability generating function of the form

$$
f(b, c ; s)=1-\frac{b}{1-c}+\frac{b s}{1-c s}, 0 \leq s \leq 1 .
$$

where $0 \leq b \leq 1,0 \leq c \leq 1$, and $b+c \leq 1$.

Proof of Corollary 3.19. Observe that the generating function of $N$ given in (2.1) is a fractional linear generating function. The results follow from equations (3.1) and (3.2) in [3] page 330 and from Theorem 3.18 .

Proof of Theorem 3.20. The result follows from the fact that the process $\left\{\mathbb{T}^{d} ; N\right\}$ dominates the process $L\left\{\mathbb{T}_{+}^{d} ; N\right\}$ and by its turn, is dominated by the process $U\left\{\mathbb{T}_{+}^{d+1} ; N\right\}$, together with Propositions 4.4 and 4.8 .

\section{REFERENCES}

[1] J.R.Artalejo, A.Economou and M.J.Lopez-Herrero. Evaluating growth measures in an immigration process subject to binomial and geometric catastrophes. Mathematical Biosciences and Engineering 4, (4), 573-594 (2007).

[2] R.Ash. Basic Probability Theory Dover ed. (2008).

[3] A.Agresti. Bounds on the Extinction Time Distribution of a Branching Process,Advances in Applied Probability, Vol. 6, No. 2, pp. 322-335 (1974). 
[4] P.Billingsley. Probability and Measure, third edition, John Wiley \& Sons. (1995).

[5] P.J.Brockwell, J.Gani and S.I.Resnick. Birth, immigration and catastrophe processes. Adv. Appl. Prob. 14, 709-731 (1982).

[6] T.E.Harris. The theory of branching processes, Dover Phoenix editions, Dover Publications, Inc., Mineola, NY, (2002).

[7] S.Kapodistria, T.Phung-Duc and J.Resing. Linear birth/immigration-death process with binomial catastrophes. Probability in the Engineering and Informational Sciences 30 (1), 79-111 (2016).

[8] J.C.D'Souza and J.D.Biggins. The supercritical Galton-Watson process in varying environments, Stochastic Process. Appl. 42 (1), 39-47, (1992).

[9] D.Stirzaker. Elementary Probability, second edition, Cambridge University Press, (2003).

[10] V.V.Junior, F.P.Machado and A. Roldán-Correa. Dispersion as a Survival Strategy. Journal of Statistical Physics, 159, (4), 937-951 (2016).

[11] F.P.Machado, A.Roldán-Correa and R.Schinazi. Colonization and Collapse. arXiv:1510.02704 (2015).

[12] R.Schinazi. Does random dispersion help survival? Journal of Statistical Physics, 159, (1), 101-107 (2015).

[13] W.Rudin. Principles of Mathematical Analysis, third edition, McGraw-Hill,Inc. (1976).

[14] H.Thorisson. Coupling, stationarity, and regeneration. Springer-Verlag, New York. (2000).

[15] A.Tucker. Applied Combinatorics $6^{\text {th }}$ ed. John Wiley 85 Sons, Inc. (2012).

(Fábio P. Machado) Institute of Mathematics and Statistics, University of São Paulo, Rua do Matão 1010, CeP 05508-090, São Paulo, SP, Brazil.

(Valdivino V. Junior) Federal University of Goias, Campus Samambaia, CEP 74001-970, GoiÂnia, GO, BraziL.

(Alejandro Roldan-Correa) Instituto de Matemáticas, Universidad de Antioquia, Calle 67, no 53-108, Medellin, Colombia

E-mail address: vvjunior@ufg.br, fmachado@ime.usp.br, alejandro.roldan@udea.edu.co 